\title{
Arbitrary energy global existence for wave equation with combined power-type nonlinearities of different signs
}

Runzhang $\mathrm{Xu}^{1 *}$, Xingchang Wang ${ }^{2}$, Huichao $\mathrm{Xu}^{1}$ and Mingyou Zhang ${ }^{2}$

\section{"Correspondence:} xurunzh@163.com

${ }^{1}$ College of Science, Harbin

Engineering University, Harbin,

150001, P.R. China

Full list of author information is

available at the end of the article

\begin{abstract}
This paper proves the global existence of solution for a class of nonlinear wave equations with nonlinear combined power-type nonlinearities of different signs for the initial data at sup-critical energy level.
\end{abstract}

Keywords: initial boundary value; wave equation; global existence; sup-critical energy

\section{Introduction}

In the present paper, we mainly consider the initial boundary value problem for the nonlinear wave equation with combined power-type nonlinearities of different signs,

$$
\begin{aligned}
& u_{t t}-\Delta u=f(u), \quad x \in \Omega, t \in[0, \infty), \\
& u(x, t)=0, \quad x \in \partial \Omega, t \in[0, \infty), \\
& u(x, 0)=u_{0}(x), \quad u_{t}(x, 0)=u_{1}(x), \quad x \in \Omega,
\end{aligned}
$$

where $\Omega \subset \mathbb{R}^{n}(n \geq 1)$ is an open bounded domain with smooth boundary $\partial \Omega, \Delta$ is the Laplace operator on $\mathbb{R}^{n}, f(u)$ is for nonlinear combined power-type nonlinearities of different signs function of $u$, i.e.

$$
f(u) \equiv \sum_{k=1}^{l} a_{k}|u|^{p_{k}-1} u-\sum_{j=1}^{s} b_{j}|u|^{q_{j}-1} u
$$

$a_{k} \geq 0,1 \leq k \leq l, b_{j}>0,1 \leq j \leq s$. In addition, $p_{k}$ and $q_{j}$ satisfy the following conditions $(\mathrm{H})$ :

$(\mathrm{H})$

$$
\begin{cases}1<q_{s}<q_{s-1} \cdots<q_{1}=q<p=p_{l}<p_{l-1}<\cdots<p_{1}<\infty & \text { if } n=1,2 \\ 1<q_{s}<q_{s-1} \cdots<q_{1}=q<p=p_{l}<p_{l-1}<\cdots<p_{1} \leq \frac{n+2}{n-2} & \text { if } n \geq 3\end{cases}
$$

Equation (1) is a class of important mathematical physical models, so there has been a lot of important work, such as [1-6], focused on it. Recently Li and Zhang [7] and Tao et

(c) The Author(s) 2016. This article is distributed under the terms of the Creative Commons Attribution 4.0 International License (http://creativecommons.org/licenses/by/4.0/), which permits unrestricted use, distribution, and reproduction in any medium, provided you give appropriate credit to the original author(s) and the source, provide a link to the Creative Commons license, and indicate if changes were made. 
al. [8] considered combined power-type nonlinearities since this kind of nonlinearities is more general than a single source term. The effects of this kind of nonlinearities on the prosperities of solution were well treated. However, the nonlinear terms considered in our paper are more general, we aim to provide some new results in this direction. This paper is a continued study of $[9,10]$, and [11], so we give a quick introduction here; for detailed background of this problem, we refer the reader to [11] and the references therein. The authors in [9] first considered problem (1)-(3) and obtained the global existence and blow up of solutions for the sub-critical case $E(0)<d$, where $E(0)$ is the initial energy and $d$ is the depth of the potential well or the mountain pass level, which will be defined later. In the same paper the critical case $E(0)=d$ was also considered and the global existence was derived. Later, Yu proved blow up of the solution for the critical case $E(0)=d$ in [10]. Furthermore, the high energy case $E(0)>0$ was treated in [11], and the blow up result was also given. Observing the above results for problem (1)-(3), helpful in the potential well method which was introduced by Payne and Sattinger [1], the global existence for the sup-critical case, i.e. $E(0)>0$, is still not solved. So the present paper solves this problem by introducing a new stable invariant set and, using the method of [12], we are focusing on proving the global existence of the solution for problem (1)-(3) in the sup-critical case $E(0)>0$.

Throughout the present paper, the following notations are used for a precise statement: $L^{p}$ denotes the space consisting of all $L^{p}$-functions on $\Omega$ with norm $\|u\|_{p}=\|u\|_{L^{p}(\Omega)},\|u\|_{2}=$ $\|u\|_{L^{2}(\Omega)}$, and the inner product $(u, v)=\int_{\Omega} u v d x$.

\section{Global existence at sup-critical case $E(0)>0$}

For problem (1)-(3) we introduce the energy functional and the Nehari functional as follows:

$$
E(t)=\frac{1}{2}\left\|u_{t}\right\|_{2}^{2}+\frac{1}{2}\|\nabla u\|_{2}^{2}-\sum_{k=1}^{l} \frac{a_{k}}{p_{k}+1}\|u\|_{p_{k}+1}^{p_{k}+1}+\sum_{j=1}^{s} \frac{b_{j}}{q_{j}+1}\|u\|_{q_{j}+1}^{q_{j}+1}
$$

and

$$
I(u)=\|\nabla u\|_{2}^{2}-\sum_{k=1}^{l} a_{k}\|u\|_{p_{k}+1}^{p_{k}+1}+\sum_{j=1}^{s} b_{j}\|u\|_{q_{j}+1}^{q_{j+1}} .
$$

Furthermore, for problem (1)-(3), we define a new stable set

$$
\mathcal{W}=\left\{u \in H_{0}^{1}(\Omega) \mid I(u)>\frac{3(p+1)}{2}\left\|u_{t}\right\|^{2}\right\}
$$

which will be used to investigate the existence of a global solution with sup-critical initial energy.

Next, we give a definition of the weak solution for problem (1)-(3).

Definition 2.1 (Weak solution [9]) We say that $u=u(x, t)$ is a weak solution of problem (1)-(3) on $\Omega \times\left[0, T_{0}\right)$ if $u \in L^{\infty}\left(0, T ; H_{0}^{1}(\Omega)\right), u_{t} \in L^{\infty}\left(0, T ; L^{2}(\Omega)\right)$ and

(i) $\left(u_{t}, v\right)+\int_{0}^{t}(\nabla u, \nabla v) d \tau=\int_{0}^{t}(f(u), v) d \tau+\left(u_{1}, v\right)$ for all $v \in H_{0}^{1}(\Omega), t \in\left(0, T_{0}\right)$;

(ii) $u(x, 0)=u_{0}(x)$ in $H_{0}^{1}(\Omega), u_{t}(x, 0)=u_{1}(x)$ in $L^{2}(\Omega)$;

(iii) $E(t)=E(0), t \in[0, T)$. 
Theorem 2.2 (Local existence [9]) Suppose that $u(x, 0) \in H_{0}^{1}(\Omega), u_{t}(x, 0) \in L^{2}(\Omega)$. Then problem (1)-(3) admits a unique local solution $u(x, t)$ defined on a maximal time interval $\left[0, T_{0}\right)$. Moreover, if

$$
\sup _{t \in\left[0, T_{0}\right)}\|u(x, t)\|_{H_{0}^{1}}<\infty
$$

then $T_{0}=\infty$.

The invariance of the stable set $\mathcal{W}$ under the flow of (1)-(3) plays an essential role while proving the global existence of the weak solution for (1)-(3). In order to obtain the invariance, we need to prove the following lemma at first.

Lemma 2.3 Let $u_{0}(x) \in H_{0}^{1}(\Omega), u_{1}(x) \in L^{2}(\Omega)$, and $u(x, t)$ be solution of problem (1)-(3) with initial data $\left(u_{0}, u_{1}\right)$. Assume that $E(0)>0$ and the initial data satisfy

$$
2\left\|u_{0}\right\|_{2}^{2}+4\left(u_{0}, u_{1}\right)+E(0)<0
$$

Then the map $\left\{t \mapsto\|u(t)\|_{2}^{2}+2\left(u, u_{t}\right)\right\}$ is strictly decreasing as long as $u(x, t) \in \mathcal{W}$.

Proof Let $F(t)=\|u(t)\|_{2}^{2}$, then

$$
\begin{aligned}
& F^{\prime}(t)=2\left(u, u_{t}\right), \\
& F^{\prime \prime}(t)=2\left(u, u_{t t}\right)+2\left\|u_{t}\right\|_{2}^{2} .
\end{aligned}
$$

Multiplying equation (1) by $u$, and integrating the obtained result with respect to $x$ over $\Omega$, we have

$$
\left(u_{t t}, u\right)+\|\nabla u\|_{2}^{2}=\sum_{k=1}^{l} a_{k}\|u\|_{p_{k}+1}^{p_{k}+1}-\sum_{j=1}^{s} b_{j}\|u\|_{q_{j}+1}^{q_{j}+1} \quad \text { for } t \in[0, \infty) .
$$

From (5) and (10), we have

$$
\left(u_{t t}, u\right)=\sum_{k=1}^{l} a_{k}\|u\|_{p_{k}+1}^{p_{k}+1}-\sum_{j=1}^{s} b_{j}\|u\|_{q_{j}+1}^{q_{j+1}}-\|\nabla u\|_{2}^{2}=-I(u) .
$$

Furthermore, from $u(t) \in \mathcal{W}$ we get

$$
F^{\prime \prime}(t)=2\left\|u_{t}\right\|_{2}^{2}-2 I(u)<0 \quad \text { for } t \in[0, \infty)
$$

which shows that $F^{\prime}(t)$ is strictly decreasing on the interval $[0, \infty)$. Obviously from $E(0)>0$ and (7), we can get

$$
F^{\prime}(0)=2\left(u_{0}, u_{1}\right)<0
$$

Then

$$
F^{\prime}(t)<F^{\prime}(0)<0 .
$$


We let $H(t)=\|u(t)\|_{2}^{2}+2\left(u, u_{t}\right)$, then

$$
H^{\prime}(t)=2\left(u, u_{t}\right)+2\left(u, u_{t t}\right)+2\left\|u_{t}\right\|_{2}^{2} .
$$

From (11), (14) becomes

$$
H^{\prime}(t)=2\left(u, u_{t}\right)-2 I(u)+2\left\|u_{t}\right\|_{2}^{2} .
$$

By (12) and (13), we have

$$
H^{\prime}(t)<0 \text { for } t \in[0,+\infty),
$$

which completes the proof.

In the following, we show the invariance of the new stable set $\mathcal{W}$ under the flow of problem (1)-(3).

Lemma 2.4 (Invariance of $\mathcal{W}$ at sup-critical case $E(0)>0$ ) Let $u_{0}(x) \in H_{0}^{1}(\Omega), u_{1}(x) \in$ $L^{2}(\Omega)$, and $u(x, t)$ be a weak solution of problem (1)-(3) with maximal existence time interval $\left[0, T_{0}\right), T_{0} \leq+\infty$. Assume that the initial data satisfy (7). Then all solutions of problem (1)-(3) with $E(0)>0$ belong to $\mathcal{W}$, provided $u_{0} \in \mathcal{W}$.

Proof We prove $u(t) \in \mathcal{W}$. Arguing by contradiction, we assume that $t_{0} \in\left(0, T_{0}\right)$ is the first time such that

$$
I\left(u\left(t_{0}\right)\right)=\frac{3(p+1)}{2}\left\|u_{t}\left(t_{0}\right)\right\|_{2}^{2}
$$

and

$$
I(u(t))>\frac{3(p+1)}{2}\left\|u_{t}(t)\right\|_{2}^{2} \quad \text { for } t \in\left[0, t_{0}\right)
$$

By (iii) in Definition 2.1, we get

$$
E(t)=E(0), \quad 0 \leq t<\infty .
$$

And by (4) and (5), we obtain

$$
\begin{aligned}
E(0)= & E\left(t_{0}\right) \\
= & \frac{1}{2}\left\|u_{t}\left(t_{0}\right)\right\|_{2}^{2}+\frac{1}{2}\left\|\nabla u\left(t_{0}\right)\right\|_{2}^{2} \\
& -\sum_{k=1}^{l} \frac{a_{k}}{p_{k}+1}\left\|u\left(t_{0}\right)\right\|_{p_{k}+1}^{p_{k}+1}+\sum_{j=1}^{s} \frac{b_{j}}{q_{j}+1}\left\|u\left(t_{0}\right)\right\|_{q_{j}+1}^{q_{j}+1} \\
\geq & \frac{1}{2}\left\|u_{t}\left(t_{0}\right)\right\|_{2}^{2}+\frac{1}{2}\left\|\nabla u\left(t_{0}\right)\right\|_{2}^{2} \\
& -\frac{1}{p+1}\left(\sum_{k=1}^{l} a_{k}\left\|u\left(t_{0}\right)\right\|_{p_{k}+1}^{p_{k}+1}-\sum_{j=1}^{s} b_{j}\left\|u\left(t_{0}\right)\right\|_{q_{j}+1}^{q_{j+1}}\right)
\end{aligned}
$$




$$
\begin{aligned}
& =\frac{1}{2}\left\|u_{t}\left(t_{0}\right)\right\|_{2}^{2}+\left(\frac{1}{2}-\frac{1}{p+1}\right)\left\|\nabla u\left(t_{0}\right)\right\|_{2}^{2}+\frac{1}{p+1} I\left(u\left(t_{0}\right)\right) \\
& \geq \frac{1}{2}\left\|u_{t}\left(t_{0}\right)\right\|_{2}^{2}+\frac{1}{p+1} I\left(u\left(t_{0}\right)\right) .
\end{aligned}
$$

Due to (15), we have

$$
E(0) \geq 2\left\|u_{t}\left(t_{0}\right)\right\|_{2}^{2}
$$

By the Cauchy-Schwarz inequality, we can get

$$
2\left\|u_{t}\left(t_{0}\right)\right\|^{2}=2\left\|u_{t}\left(t_{0}\right)+u\left(t_{0}\right)\right\|^{2}-2\left\|u\left(t_{0}\right)\right\|^{2}-4\left(u\left(t_{0}\right), u_{t}\left(t_{0}\right)\right)
$$

From (19) and Lemma 2.3, (18) becomes

$$
\begin{aligned}
E(0) & \geq 2\left\|u_{t}\left(t_{0}\right)+u\left(t_{0}\right)\right\|^{2}-2\left\|u\left(t_{0}\right)\right\|^{2}-4\left(u\left(t_{0}\right), u_{t}\left(t_{0}\right)\right) \\
& \geq-2\left(\left\|u\left(t_{0}\right)\right\|^{2}+2\left(u\left(t_{0}\right), u_{t}\left(t_{0}\right)\right)\right) \\
& \geq-2\left(\left\|u_{0}\right\|^{2}+2\left(u_{0}, u_{1}\right)\right) \\
& =-2\left\|u_{0}\right\|^{2}-4\left(u_{0}, u_{1}\right),
\end{aligned}
$$

that is,

$$
2\left\|u_{0}\right\|^{2}+4\left(u_{0}, u_{1}\right)+E(0) \geq 0 .
$$

It is obvious that (7) contradicts (21). This completes the proof.

Theorem 2.5 Let $u_{0}(x) \in H_{0}^{1}(\Omega), u_{1}(x) \in L^{2}(\Omega)$, and let $u(x, t)$ be a weak solution of problem (1)-(3) with maximal existence time interval [0, $\left.T_{0}\right), T_{0} \leq+\infty$. Assume that $E(0)>0$, $u_{0} \in \mathcal{W}$, and (7) holds, then the solution of problem (1)-(3) exists globally.

Proof Let $u(t)$ be the weak solution of problem (1)-(3) with $E(0)>0$ and $u_{0} \in \mathcal{W}$. Then from Lemma 2.4, we have $u(x, t) \in \mathcal{W}$, that is,

$$
I(u(t))>\frac{3(p+1)}{2}\left\|u_{t}(t)\right\|^{2} \quad \text { for } t \in\left[0, T_{0}\right) .
$$

Therefore by (4), (5), and (22), we get

$$
\begin{aligned}
E(0)= & E(t) \\
= & \frac{1}{2}\left\|u_{t}(t)\right\|_{2}^{2}+\frac{1}{2}\|\nabla u(t)\|_{2}^{2} \\
& -\sum_{k=1}^{l} \frac{a_{k}}{p_{k}+1}\|u(t)\|_{p_{k}+1}^{p_{k}+1}+\sum_{j=1}^{s} \frac{b_{j}}{q_{j}+1}\|u(t)\|_{q_{j}+1}^{q_{j}+1} \\
\geq & \frac{1}{2}\left\|u_{t}(t)\right\|_{2}^{2}+\left(\frac{1}{2}-\frac{1}{p+1}\right)\|\nabla u(t)\|_{2}^{2}+\frac{1}{p+1} I(u(t)) \\
> & 2\left\|u_{t}(t)\right\|_{2}^{2}+\left(\frac{1}{2}-\frac{1}{p+1}\right)\|\nabla u(t)\|_{2}^{2},
\end{aligned}
$$


which implies

$$
\begin{aligned}
& u(x, t) \quad \text { is bounded in } L^{\infty}\left(0, T_{0} ; H_{0}^{1}(\Omega)\right), \\
& u_{t}(x, t) \text { is bounded in } L^{\infty}\left(0, T_{0} ; L^{2}(\Omega)\right) .
\end{aligned}
$$

Hence from Theorem 2.2, it follows that $T_{0}=\infty$ and the solution of problem (1)-(3) exists globally.

\section{Competing interests}

The authors declare that they have no competing interests.

\section{Authors' contributions}

The work presented here was carried out in collaboration between all authors. RX found the motivation of this paper and suggested the outline of the proofs. HX and MZ provided many good ideas for completing this paper. XW finished the proof of the main theorem. All authors have contributed to, read and approved the manuscript.

\section{Author details}

${ }^{1}$ College of Science, Harbin Engineering University, Harbin, 150001, P.R. China. ${ }^{2}$ College of Automation, Harbin Engineering University, Harbin, 150001, P.R. China.

\section{Acknowledgements}

This work was supported by the National Natural Science Foundation of China (11471087), the China Postdoctoral Science Foundation (2013M540270), the Heilongjiang Postdoctoral Foundation (LBH-Z13056), the Support Plan for the Young College Academic Backbone of Heilongjiang Province (1252G020), the Fundamental Research Funds for the Central Universities. Dr. Xu Runzhang also thanks Prof. Yue Liu for his invitation of visit to UTA. The authors appreciate Prof. Weike Wang for his valuable suggestions.

\section{Received: 26 May 2016 Accepted: 22 November 2016 Published online: 01 December 2016}

\section{References}

1. Payne, LE, Sattinger, DH: Saddle points and instability of nonlinear hyperbolic equations. Isr. J. Math. 22, 273-303 (1975)

2. Gazzola, F, Squassina, M: Global solutions and finite time blow up for damped semilinear wave equations. Ann. Inst. Henri Poincaré, Anal. Non Linéaire 23, 185-207 (2006)

3. Ikehata, R: Some remarks on the wave equations with nonlinear damping and source terms. Nonlinear Anal. 27 1165-1175 (1996)

4. Cavalcanti, MM, Domingos Cavalcanti, VN, Martinez, P: Existence and decay rate estimates for the wave equation with nonlinear boundary damping and source term. J. Differ. Equ. 203, 119-158 (2004)

5. Cavalcanti, MM, Domingos Cavalcanti, VN, Lasiecka, I: Well-posedness and optimal decay rates for the wave equation with nonlinear boundary damping-source interaction. J. Differ. Equ. 236, 407-459 (2007)

6. Alves, CO, Cavalcanti, MM: On existence, uniform decay rates and blow up for solutions of the 2-D wave equation with exponential source. Calc. Var. Partial Differ. Equ. 34(3), 377-411 (2009)

7. Li, K, Zhang, Q: Existence and nonexistence of global solutions for the equation of dislocation of crystals. J. Differ. Equ. $146,5-21(1998)$

8. Tao, T, Visan, M, Zhang, X: The nonlinear Schrödinger equation with combined power-type nonlinearities. Commun. Partial Differ. Equ. 32, 1281-1343 (2007)

9. Liu, Y, Xu, R: Wave equations and reaction-diffusion equations with several nonlinear source terms of different sign. Discrete Contin. Dyn. Syst., Ser. B 7, 171-189 (2007)

10. Yu, T, Tang, L, Liu, B, Xu, R: Wave equations and reaction-diffusion equations with several nonlinear source terms with critical energy. AIP Conf. Proc. 1479, 2435-2438 (2012)

11. Shen, J, Xu, R, Yang, Y, Chen, S, Su, J, Huang, S: Nonlinear wave equations and reaction-diffusion equations with several nonlinear source terms of different signs at high energy level. ANZIAM J. 54, 153-170 (2013)

12. Yang, Y, Shen, J, Xu, R: Global existence of solutions for 1-D nonlinear wave equation of sixth order at high initial energy level. Bound. Value Probl. 2014, 31 (2014) 